\title{
Wavelength-selective components in SOI photonic wires fabricated with deep UV lithography
}

\author{
Pieter Dumon*, Wim Bogaerts*, Dries Van Thourhout*, Dirk Taillaert*, \\ Vincent Wiaux ${ }^{\dagger}$, Stephan Beckx ${ }^{\dagger}$, Johan Wouters ${ }^{\dagger}$ and Roel Baets* \\ ${ }^{*}$ Ghent University - IMEC, Department of Information Technology \\ Sint-Pietersnieuwstraat 41, 9000 Gent, BELGIUM \\ Email: pieter.dumon@intec.UGent.be \\ ${ }^{\dagger}$ IMEC v.z.w., Silicon Process Technology Division \\ Kapeldreef 75, 3001 Leuven, BELGIUM
}

\begin{abstract}
We demonstrate both ring resonator drop filters and arrayed waveguide gratings in Silicon-on-insulator photonic wires. The structures are fabricated in a CMOS line using deep UV lithography and dry etching processes. Waveguide losses are as low as $2.4 \mathrm{~dB} / \mathrm{cm}$ for a $500 \mathrm{~nm}$ wide photonic wire, with excess bend losses of $0.03 \mathrm{~dB} / 90^{\circ}$ in a $3 \mu \mathrm{m}$ bend. Using grating fiber couplers for measurements, we show ring and racetrack resonators with a $Q$ up to 12700 and an 8-channel arrayed waveguide grating with a footprint of about $0.1 \mathrm{~mm}^{2}, 3 \mathrm{~nm}$ channel spacing and $-6 d B$ crosstalk between channels.
\end{abstract}

\section{INTRODUCTION}

For long-distance optical-fiber communication, signals are transmitted using a number of channels with carrier wavelengths. This Wavelength Division Multiplexing (WDM) requires components to combine and/or separate the channels at the input and output of the fibre. There are several approaches to such wavelength-selective functions. Cavities can extract a single channel from an optical waveguide and/or insert another signal. [1]. Alternatively, an Arrayed Waveguide Grating (AWG) uses a distributed circuit of various delay lines to sepatare/combine different wavelength channels [2].

Today's commercially available wavelength (de)multiplexers have footprints of tens of $\mathrm{cm}^{2}$, mainly because the low-index-contrast waveguides require large bend radii to keep light confined and suppress radiation losses. To reduce the chip area and increase integration, waveguides with sharp bends are a necessity. The confinement can be increased by using a higher refractive index contrast between core and (side) cladding, but to keep the waveguide single-mode, it has to be made narrower. In semiconductor, with a refractive index of 3 or higher, the threshold width is of the order of $600 \mathrm{~nm}$. Such high-contrast, submicron waveguides are also called photonic wires.

We present waveguides and wavelength-selective components in Silicon-on-insulator (SOI). In SOI, the Silicon core is shielded from the Silicon substrate by an $\mathrm{SiO}_{2}$ cladding (buffer) layer. One advantage of SOI is its compatibility with CMOS fabrication processes, which allowed us to make use of advanced CMOS technology, including deep UV lithography.

We will first explain some basic properties of both ring resonators and arrayed waveguide gratings. Then we will give

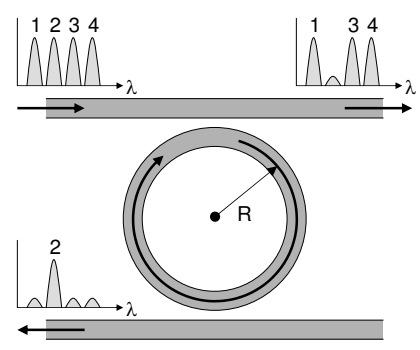

(a)

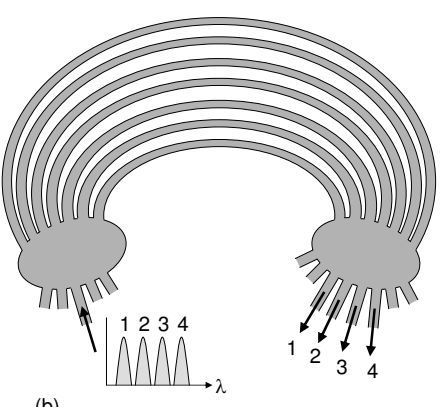

(b)
Fig. 1. (a) Ring resonator, (b) Arrayed waveguide grating (AWG)

some details on the fabrication technique and the measurement setup. Finally, we will discuss a number of measurement results.

\section{WAVELENGTH-SELECTIVE WAVEGUide Components}

In WDM systems, information is transmitted in channels, encoded as a modulation of a carrier wave at a certain wavelength. This results in a number of wavelength channels which occupy a spectral band around each carrier wavelength.

\section{A. Ring Resonators}

To select a given channel from the compound signal, one can use a filter to extract one channel, like the ring resonatorbased drop filter shown in Fig. 1a. In a ring resonator, power is passed from the input waveguide to the output waveguide only for those wavelengths near the resonance wavelength, i.e. when the wavelength fits a whole number of times inside the circumference of the ring [1].

The quality of a ring resonator, expressed by its $Q$-factor, is directly determined by the losses. Typically, a $Q$ of over 1000 is required for coarse WDM applications. The losses of the ring should therefore be kept under control.

The free spectral range (FSR) of the ring is the separation between two resonant wavelengths. When using a WDM system with a large number of channels, the FSR of the ring must be sufficiently large to select only a single channel. As the FSR is inversely proportional to the circumference length, 


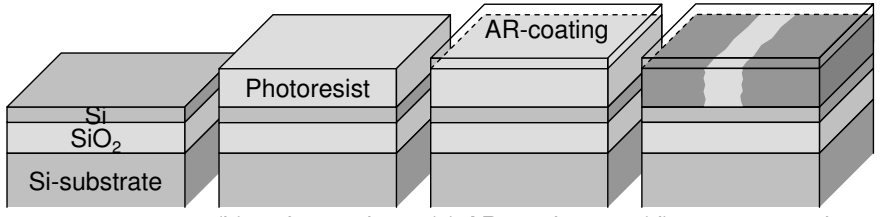

(a) bare wafer

(b) resist coating and soft bake

(c) AR coating

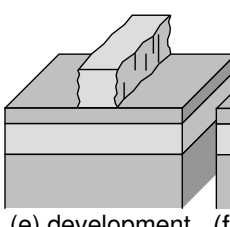

(e) development

(d) exposure and post-bake
Fig. 2. The fabrication process process for nanophotonic waveguide structures in SOI.

the ring should therefore be sufficiently small. This is, again, a plea for waveguides with sharp bends.

\section{B. Arrayed Waveguide Gratings (AWG)}

Instead of dropping a single channel at a time, one can also demultiplex all channels simultaneously. In an AWG (figure 1b), a multi-channel signal is fed into a number of delay lines [2]. The ends of the delay lines act as a phased array antenna, refocusing the light. As the phase delay differs for each wavelength, so does the focal point, so one can extract each wavelength channel into its own waveguide.

\section{FABRICATION}

For our high-contrast waveguides, we used Silicon-onInsulator (SOI) purchased from SOITEC. The top Silicon layer has a thickness of $220 \mathrm{~nm}$ and the oxide layer $1 \mu \mathrm{m}$ thick. The actual processing was carried out in the advanced CMOS research environment of IMEC, Belgium. The fabrication process, illustrated in Fig. 2, is described in detail in [3], [4]. After applying and baking the Shipley UV3 photoresist, a deep UV stepper with an illumination wavelength $248 \mathrm{~nm}$ is used to define the patterns. After another baking step the resist is developed. To smoothen the resist patterns and decrease the line width of the waveguides, a "resist hardening" plasma treatment is applied to the developed photoresist. This is then used directly as an etch mask for the Silicon etch, which uses a ICP-RIE etch technique with a $\mathrm{Cl}_{2} / \mathrm{HBr} / \mathrm{He} / \mathrm{O}_{2}$ chemistry. Finally, the remainder of the resist is removed.

\section{Measurement Technique}

To characterize the fabricated structures, we measured the transmission in the wavelength range between $1500 \mathrm{~nm}$ and $1600 \mathrm{~nm}$. To facilitate incoupling and outcoupling of light, we used grating-based fiber couplers, which couple light from a single mode fiber to a $10 \mu \mathrm{m}$ wide ridge waveguide with over $20 \%$ efficiency [4], [5]. The principle is shown in Fig. 3. Light is coupled in from a slightly tilted fiber by the grating coupler. The broad ridge waveguide is adiabatically tapered down to a single mode waveguide, which is used to feed light into

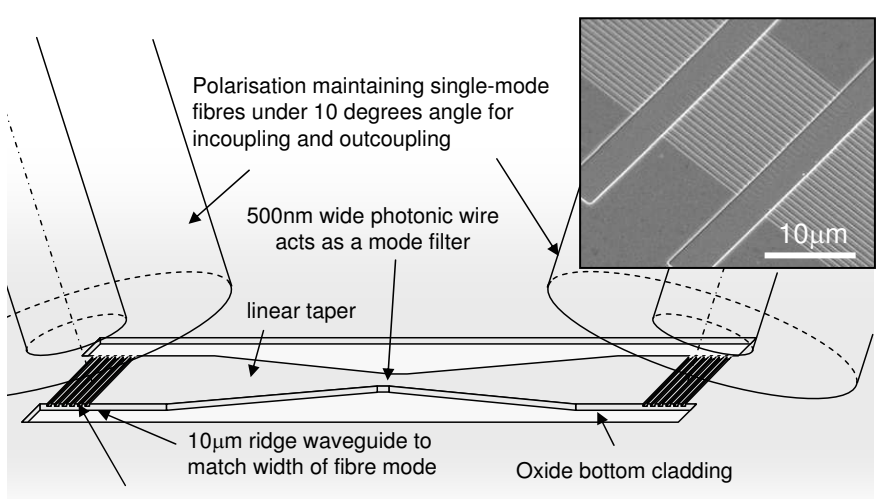

Shallow-etched fibre coupler grating

for coupling to single-mode fibre

Fig. 3. Measurement of nanophotonic waveguide components using a grating coupler to single-mode fiber. The inset shows an SEM of the grating coupler

the various components on the chip. at each output port of the component, a similar waveguide is tapered out to a $10 \mu \mathrm{m}$ width and the light is coupled back to the fiber by an identical grating.

The efficiency of the grating coupler has a Gaussian wavelength dependence [4], [5]. The couplers used here have a peak efficiency of $18 \%$ at a wavelength of $1540 \mathrm{~nm}$ and a $3 d B$ bandwidth of $56 \mathrm{~nm}$.

\section{Measurement Results}

We fabricated a large number of test structures to characterize the various aspects of photonic wire-based components. Some examples are shown in Fig. 4.

\section{A. Waveguide Losses and Bends}

Low-loss waveguides are a prerequisite for both ring resonators and AWGs. In the structures we fabricated, there are two dominant loss mechanisms. Because of the finite thickness of the oxide cladding, light can leak out towards the Silicon substrate. These losses can be reduced by using a thicker oxide cladding layer. The second loss mechanism comes from scattering at imperfections on the etched sidewall of the waveguide. Good fabrication technology is needed to keep these losses to a minimum. We have already reported very low propagation losses of $2.4 \mathrm{~dB} / \mathrm{cm}$ for straight SOI waveguides [4], [6].

In a waveguide bend, there can be additional losses due to radiation. These losses will increase with sharper bends.

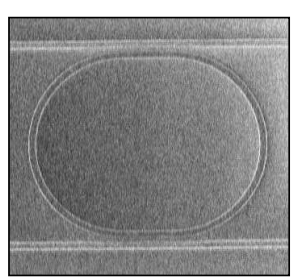

(a)

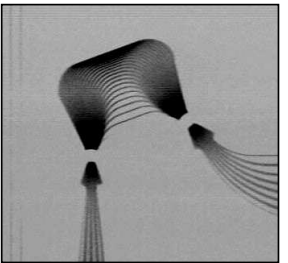

(b)

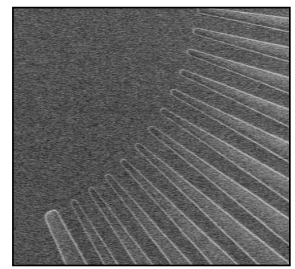

(c)
Fig. 4. Micrograph of SOI waveguide structures. (a) ring resonator, (b) an Arrayed Waveguide Grating, (c) detail of the star-coupler of the AWG. 


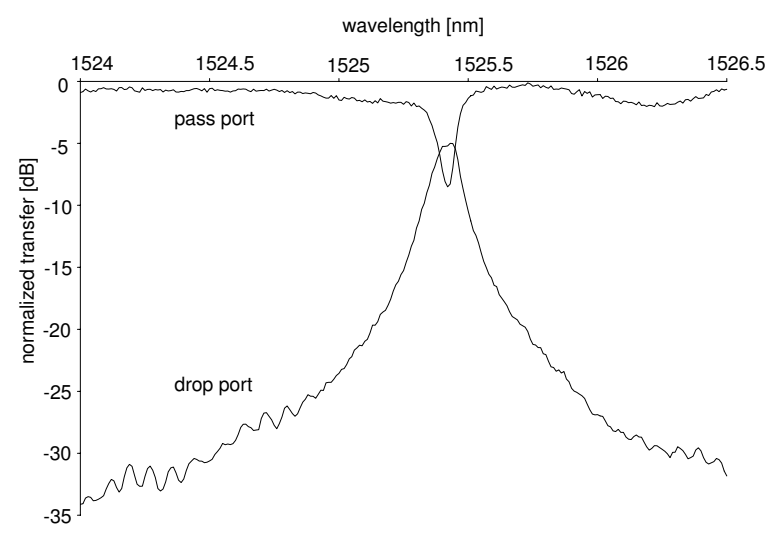

Fig. 5. Transmission of the pass and drop port of the racetrack resonator in Fig. 4 normalized to the transmission of a straight waveguide.

We have characterized losses of bends by measuring the transmission of curled-up waveguides with various numbers of bends and bend radii. For waveguides with a width of around $500 \mathrm{~nm}$ we measured excess bend losses (with respect to the straight waveguide) of around $0.03 \mathrm{~dB} / 90^{\circ}$ for bend radii of $3 \mu m$.

\section{B. Compact Ring resonators}

With these low bend losses, ring and racetrack resonators become possible. Fig. 4a shows a racetrack resonator with a bend radius of $4 \mu \mathrm{m}$ and a straight coupling section of $3.14 \mu \mathrm{m}$. The transmission of both pass port and drop port is plotted in Fig. 5. The resonator has a $Q$ of 12700 with a FWHM of $12 \mathrm{~nm}$ around $1525 \mathrm{~nm}$. The free spectral range is $16.5 \mathrm{~nm}$, resulting in a finesse of 137 . However, there is still significant add/drop crosstalk. We have also demonstrated racetrack resonators with a lower $Q$ of 3000 but a higher coupling efficiency and therefore lower crosstalk [6]

\section{Arrayed Waveguide Gratings (AWGs)}

We have designed and fabricated an 8-channel AWG, illustrated in Fig. $4 \mathrm{~b}$ and $4 \mathrm{c}$. This device has a footprint of $380 \mu \mathrm{m} \times 290 \mu \mathrm{m}$, or about $0.1 \mathrm{~mm}^{2}$. The output of the 8 output waveguides is plotted in Fig. 6. The insertion loss due to the star coupling sections is approximately $-8 d B$. The channel spacing is $3 \mathrm{~nm}$, with a free spectral range of $24 \mathrm{~nm}$. However, the crosstalk is still signifcant, between $-6 d B$ and $-9 B$.

\section{CONCLUSION}

We have fabricated wavelength-selective components based on ring resonators and arrayed waveguide gratings in Siliconon-insulator. For the fabrication we made use of standard CMOS processes based on deep UV lithography. For easy measurements, we use grating-based fiber couplers to couple light from and to a single-mode fiber.

Waveguide losses for single-mode straight photonic wires are as low as $2.4 \mathrm{~dB} / \mathrm{cm}$. For bends, we have measured excess losses of $0.03 d B / 90^{\circ}$ in $3 \mu \mathrm{m}$ bend. We have also demonstrated racetrack resonators with a $Q$ of over 12000 and a coupling efficiency of $-5 d B$.

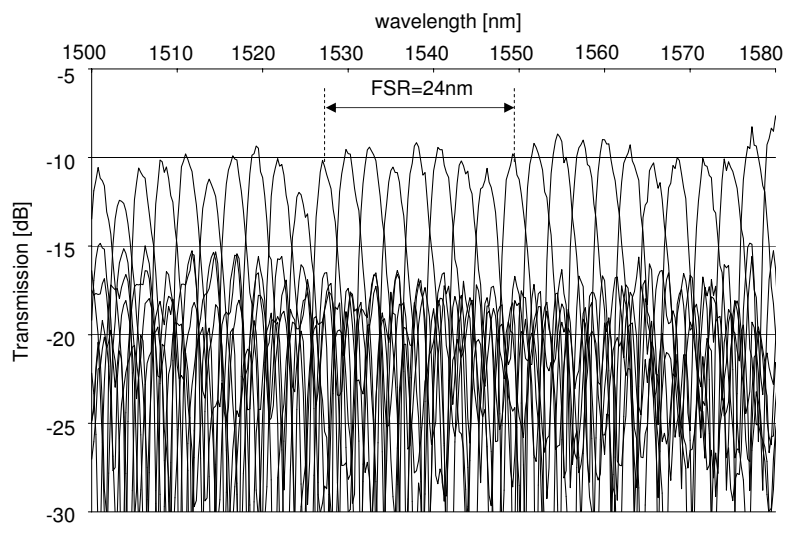

Fig. 6. Transmission of the 8 output channels of the AWG in Fig. 4b.

We have also measured an 8-channel arrayed waveguide grating with $3 \mathrm{~nm}$ channel spacing. Insertion losses are $8 d B$ and the crosstalk between adjacent channels is $-6 d B$ or better.

\section{ACKNOWLEDGMENT}

Part of this work was supported by the European Union through the IST-PICCO project.

Part of this work was carried out in the context of the Belgian IAP-PHOTONnetwork project.

P. Dumon and W. Bogaerts thank the Flemish Institute for the industrial advancement of scientific and technological Research (IWT) for a specialization grant. D. Van Thourhout acknowledges the Belgian Federal Office for Scientific, Technical and Cultural Affairs for financial support.

The authors would also like to thank D. Vangoidsenhoven for the wafer exposures, Rudi de Ruyter and Johan Mees for their work on the mask design, and Johan Wouters for the extensive work on the etch development. Also many thanks to the people of the P-Line for the processing.

\section{REFERENCES}

[1] M. Chin and T. Ho, "Design and modeling of waveguide-coupled singlemode microring resonators," J. Lightwave Technol., vol. 16, no. 8, pp. 1433-1446, 1998.

[2] C. Dragone, "An NxN optical multiplexer using a planar arrangement of two star couplers," IEEE Photon. Technol. Lett., vol. 3, no. 9, pp. 812-814, Sept. 1991

[3] W. Bogaerts, R. Baets, P. Dumon, V. Wiaux, S. Beckx, D. Taillaert, B. Luyssaert, J. Van Campenhout, P. Bienstman, and D. Van Thourhout, "Nanophotonic waveguides in Silicon-on-insulator fabricated with CMOS technology," accepted for publication in J. Lightwave Technol., 2004.

[4] W. Bogaerts, D. Taillaert, B. Luyssaert, P. Dumon, J. Van Campenhout, P. Bienstman, D. Van Thourhout, R. Baets, V. Wiaux, and S. Beckx, "Basic structures for photonic integrated circuits in Silicon-on-insulator," Opt. Expr., vol. 12, no. 8, pp. 1583-1591, April 2004.

[5] D. Taillaert, W. Bogaerts, P. Bienstman, T. Krauss, P. Van Daele, I. Moerman, S. Verstuyft, K. De Mesel, and R. Baets, "An out-of-plane grating coupler for efficient butt-coupling between compact planar waveguides and single-mode fibers," IEEE J. Quant. Electron., vol. 38, no. 7, pp. 949-955, 2002.

[6] P. Dumon, W. Bogaerts, V. Wiaux, J. Wouters, S. Beckx, J. Van Campenhout, D. Taillaert, B. Luyssaert, P. Bienstman, D. Van Thourhout, and R. Baets, "Low-loss SOI photonic wires and ring resonators fabricated with deep UV lithography," IEEE Photon. Technol. Lett., vol. 16, no. 5, pp. 1328-1330, May 2004. 